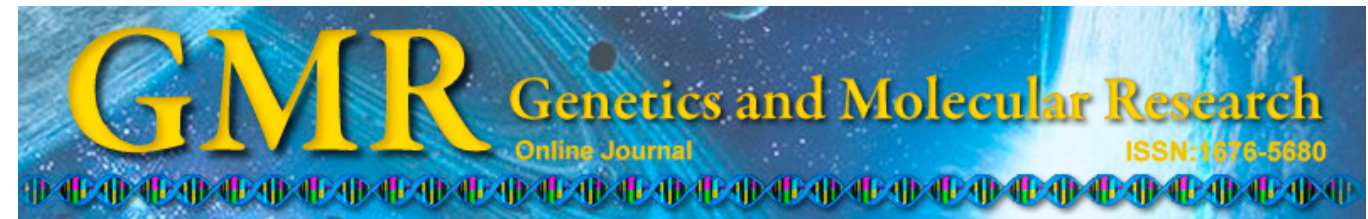

\title{
Molecular characterization and tissue-specific expression of invariant chain isoform in Muscovy Duck (Cairina moschata)
}

\author{
S.J. Liu', ${ }^{1,2}$ F.F. Chen ${ }^{2}$, C. Wu ${ }^{2}$, Q.S. Ni ${ }^{2}$ and W.Y. Yu ${ }^{2}$ \\ ${ }^{1}$ School of Life Science, Fuyang Teachers' College of Anhui Province, \\ Fuyang, China \\ ${ }^{2}$ Key Laboratory of Zoonoses of Anhui Province, Anhui Agricultural University, \\ Hefei, China \\ Corresponding author: W.Y. Yu \\ E-mail: yuweiyi@ahau.edu.cn
}

Genet. Mol. Res. 13 (4): 8971-8981 (2014)

Received January 9, 2014

Accepted May 28, 2014

Published October 31, 2014

DOI http://dx.doi.org/10.4238/2014.October.31.12

\begin{abstract}
Invariant chain (Ii) isoform, through its thyroglobulin-like (Tg) domain, inhibits cysteine proteases during antigen presentation in vertebrates. In birds, the Ii of Muscovy Duck (MDIi) has 2 forms: MDIi1 and MDIi-2 (MDIi isoform). To understand the genetic information and expression characteristics of MDIi-2, polymerase chain reaction, and bioinformatic analysis were performed for $M D I i-2$ from healthy adult Muscovy Duck. The full-length MDIi-2 cDNA sequence was found to be 1377-base pairs, encoding a 285-amino acid protein. MDIi2 contains 63 amino acids with an insertion sequence in the Tg domain. MDIi-2 shares high identity (72.51-94.74\%) with the same protein in other birds. The Tg domain of MDIi-2 is highly conserved and showed relatively high identity (96.83\%) among all tested birds. The molecular structure of the Tg domain supports this conservation. MDIi-2 expression was measured in various tissues using real-time quantitative polymerase chain reaction. Similar to $M D I i-1, M D I i-2$ was detected in all tissues but at different levels. Higher expression level was observed
\end{abstract}


in the spleen, intestinal mucosa, and bursa stipe (bursa of Fabricius stipe) than in other tissues. This suggests that MDIi-2, like MDIi-1, plays an essential role in all tissues and that its differential expression may be related to its functions in these tissues. The coexistence of 2 MDIi isoforms indicates that their functions are correlated in Muscovy Duck. This study improves the understanding of poultry immunology and may be used to improve measures to protect Muscovy Duck from disease.

Key words: Clone; Differential expression; Invariant chain isoform; Muscovy Duck; Real-time quantitative PCR

\section{INTRODUCTION}

The invariant chain (Ii) plays an important role as a chaperone in major histocompatibility complex (MHC) class II molecules by properly guiding the MHC II heterodimer through the cell, peptide loading, and presenting the antigen on the surface of an antigenpresenting cell (Arunachalam et al., 1994; Bergmann, 2012). Ii guides antigen presentation through its $\mathrm{N}$-terminal sorting signal (Landsverk et al., 2009; Xu et al., 2013). Thus, Ii has been used as immune carrier (Chen and Yu, 2010; Wälchli et al., 2014) in the construction of genetic vaccines, in which the class II-associated Ii-derived peptide domain was replaced with sequences encoding for specific antigen epitopes. Genetic vaccines containing Ii promote significantly greater immune responses than DNA vaccines encoding the Ii alone or antigen epitope alone both in vitro and in vivo (Hung et al., 2007; Nagaraj et al., 2008; Holst et al., 2011; Walline et al., 2014).

There are 4 isoforms of the human invariant chain, including p33, p35, p41, and $\mathrm{p} 43$, which are generated through a combination of alternative translation initiation and splicing events for a single gene (Strubin et al., 1986). p33 is the predominant isoform (Warmerdam et al., 1996; Bergmann, 2012) and forms a complex with MHC II to drive antigen presentation. p41 and p43 contain a thyroglobulin-like (Tg) domain encoded by exon $6 \mathrm{~b}$ of the Ii gene (Gedde-Dahl et al., 1997). The Tg domain is a cysteine-rich stretch of 64 amino acids and displays highly homology to a repetitive sequence of thyroglobulin (a precursor of thyroid hormone) near the C-terminal region of Ii (O'Sullivan et al., 1987). The $\mathrm{Tg}$ domain is a structural motif and inhibits cysteine proteases during antigen-presentation (Reich et al., 2011; Criscitiello et al., 2012). Although p33 and p41 have different structures and functions, each is involved in the antigen presenting process (Criscitiello et al., 2012). Since genetic vaccines containing Ii is constructed on antigen-presentation mechanism of Ii, the Ii isoform might be involved in the the antigen presenting process of the genetic vaccines. Thus, an increased understanding of the Ii isoform may be benefit for improving genetic vaccines containing Ii.

Only two isoform forms of Ii, p31 and p41, which are similar to human p33 and p41, have been discovered in rat and some birds (Yamamoto et al., 1985; Zhong et al., 2006; Chen et al., 2011). p41 also consistently contains conserved Tg domains, which are remarkably similar each other on the amino acid sequence level and in their molecular structures in various species (Zhong et al., 2006; Chen et al., 2013). Ii in Muscovy Duck (MDIi) might 
contains these 2 isoforms, MDIi-1 [MDIi in reference (Liu et al., 2011), GenBank accession No. HQ909102; KF584915.1 referred to as MDIi-1 for convenience], which has been cloned previously, and MDIi-2. However, genetic information and the expression characteristics of the presumed isoform $M D I i-2$ have not been described. In this study, we identified $M D I i-2$ by amplification and bioinformatic analysis for a fragment of the Tg insertion sequence. Relative quantitative real-time polymerase chain reaction (real-time RQ-PCR) was also used to examine the different expression of MDIi-2 in various tissues of Muscovy Duck. Our results may increase the understanding of poultry immunology and KF584915.1may be used to improve the protection of Muscovy Duck against diseases.

\section{MATERIAL AND METHODS}

\section{Sampling from Muscovy Duck, RNA isolation, and cDNA synthesis}

Samples of blood, brain, bursa fundus (bursa of Fabricius fundus), bursa stipe (bursa of Fabricius stipe), heart, intestinal mucosa, kidney, liver, lung, skeletal muscle, spleen, and thymus were collected from healthy adult Muscovy Ducks at approximately 5 months of age. Ducks were purchased from a poultry farm in the suburban district of Hefei City, China. The methods used to extract total RNA from tissues and those for single-stranded cDNA synthesis from $5 \mu \mathrm{g}$ RNA have been reported previously (Liu et al., 2011).

\section{PCR amplification of the Tg domain of MDIi-2}

The MDIi-1 gene (GenBank accession No. HQ909102) was aligned with the Ii isoforms (GenBank accession No. goose, HM636053.1; quail, HQ130789.1; chicken, AY597054.1) containing $\mathrm{Tg}$ domains for selected related species using the DNAMAN version 6.0 software (Lynnon BioSoft, Quebec, Canada). The insertion site of the Tg domain was found to potentially reside in MDIi-1 between 623-624 base pairs (bp), with a length of approximately $190 \mathrm{bp}$. The primer pair 2-Tg-F and 2-Tg-R (Table 1) was designed outside of the Tg insertion site in MDIi-1 to amplify the Tg sequence. The length of amplification products were approximately $100 \mathrm{bp}$ (no $\mathrm{Tg}$ sequence) or $290 \mathrm{bp}$ (with $\mathrm{Tg}$ sequence). Amplification products were purified from 1\% agarose gels using a gel extraction kit (BioTek Instruments, Inc., Winooski, VT, USA). The purified fragment was cloned into the pMD-18T vector (TaKaRa, Shiga, Japan) and sequenced by Sangon Biotech Co. Ltd. (Shanghai, China).

\begin{tabular}{|c|c|c|}
\hline Application & Primer & Sequence (5' to $3^{\prime}$ ) \\
\hline \multirow{2}{*}{ MDIi-2 Tg PCR } & $2-\mathrm{Tg}-\mathrm{F}$ & CGGACGAGCGTAAAGCCATC \\
\hline & $2-\mathrm{Tg}-\mathrm{R}$ & TCAGCGCCCAGCTTGAGCATG \\
\hline \multirow[t]{2}{*}{ Full-length PCR } & Full-Ii-F & TGGGGCAGCCATGGCCGAGGAG \\
\hline & Full-Ii-R & TCCTTCACACAACGCCCTCACT \\
\hline \multirow[t]{4}{*}{ Real-time PCR } & 2-RTP-F & GCGGAGAAAGTGCAAACTAAGTG \\
\hline & 2-RTP-R & TGTCCACCCCGGAGAAGATCATC \\
\hline & $\beta$-actin-F & ATGTGGATCAGCAAGCAGGA \\
\hline & $\beta$-actin-R & ACAAGGGTGTGGGTGTTGG \\
\hline
\end{tabular}

Full-length PCR $=$ PCR for obtaining the fragments including most partial sequence of full-length invariant chain isoforms of muscovy duck ( $M D I i$ isoform) $\mathrm{cDNA} ; \mathrm{F}=$ forward primer; $\mathrm{R}=$ reverse primer. 


\section{Sequence assembly and verification}

The deduced full-length MDIi-2 cDNA sequence was assembled using the full-length MDIi-1 cDNA sequence (GenBank accession No. HQ909102) and the amplified sequence of Tg using the DNAMAN software. To confirm the assembled full-length MDIi-2 sequence, the full-length primer pair Full-Ii-F and Full-Ii-R (Table 1) were designed based on full-length $M D I i-2$. Next, the assembled sequence was verified by sequencing the fragment that had been amplified with the primer Full-Ii-F and Full-Ii-R and subjected to homology analysis.

\section{Bioinformatic analysis and homology modeling}

The methods used for bioinformatic analysis and homology modeling have been reported previously (Liu et al., 2011). Briefly, the cDNA sequences of MDIi-2 were analyzed using DNASTAR 5.0 (DNASTAR Inc., Madison, WI, USA). Similarity analysis was performed using the BLAST algorithm at NCBI (http://www.ncbi.nlm.nih.gov/BLAST) and the DNAMAN version 6.0 software. Multiple alignments of the MDIi-2 proteins were performed using the Clustal W multiple alignment programs (http://www.ebi.ac.uk/clustalw/). The structure model of Ii was obtained using the Expasy's translate tool (http://swissmodel.expasy.org/). Theoretical models were displayed and analyzed using the RasMol software (http://www.RasMol.org).

\section{Real-time RQ-PCR}

Differential expression of MDIi-2 in 12 tissues was detected by real-time RQ-PCR using the primer pair 2-RTP-F and 2-RTP-R (Table 1), which were designed based on the Tg sequences from the confirmed MDIi-2. Additionally, $\beta$-actin-F and $\beta$-actin-R primers (Table 1) were designed to amplify the duck $\beta$-actin gene as a internal control (GenBank accession No. GU564232). Real-time RQ-PCR amplification of MDIi-2 was carried; MDIi-1 was also amplified as reported in reference previous study (Liu et al., 2011), using the same methods and cDNA from 12 tissues, under the same conditions, on the same instruments. The lowest value of expression level of MDIi-2 in the tissue was used as calibrator. One-way analysis of variance was used for statistical analysis. $\mathrm{P}<0.01$ was considered to be statistically significant.

\section{RESULTS}

\section{Identification of $\mathrm{Tg}$ and $\mathrm{MDIi}-2$ sequences}

The amplification products of the $\mathrm{Tg}$ fragment from Muscovy Duck were analyzed on $1 \%$ agarose gels (Figure 1). Two nucleic acid bands (first lane), approximately 100 and 290 bp, were of the expected sizes, but the abundance of the 290-bp band determined to be the target fragment containing the Tg domain was clearly lower than that of the 100-bp band. After sequencing, the target fragment was verified to be a partial fragment of Ii according to NCBI BLAST analysis. A 189-bp conserved sequence contained the Tg domain. The deduced fulllength MDIi-2 cDNA was obtained by splicing and assembling the $T g$ fragment with MDIi-1. The full-length MDIi-2 cDNA was verified by full-length PCR (data not shown). MDIi-2 (GenBank accession No. KF584915) is 1377 bp (Figure 2), including an 858-bp open reading frame located between nucleotides 35 and 892, a 34-bp 5'-terminal untranslated region, and 
a 485-bp 3'-untranslated region followed by a 12-bp poly (A) tail. The $\mathrm{Tg}$ domain resides in MDIi-2 between 624-812 bp; the length of the Tg sequence is $189 \mathrm{bp}$ (underlined in Figure 2).

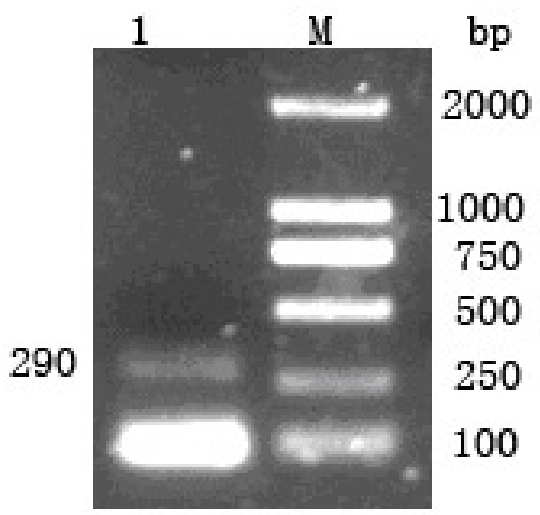

Figure 1. Amplification products of Tg fragments of $M D I i-2$. Lane $1=$ amplification products of $\mathrm{Tg}$ fragments; lane $M=$ standard marker of nucleic acids sizes.

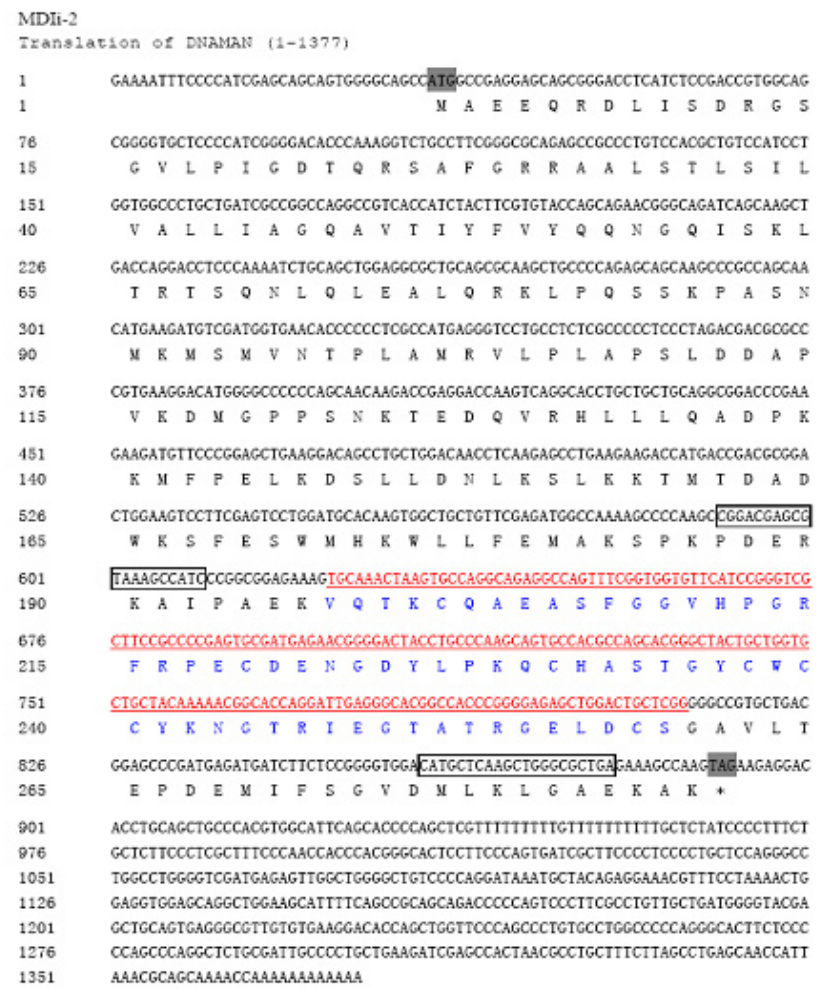

Figure 2. Confirmed cDNA sequence of MDIi-2. The codons ATG and TAG shaded in gray are the initiation and termination codons, respectively. The nucleic acid sequences of the $\mathrm{Tg}$ domain are marked in red and with a single underline, while the amino acid sequences of the Tg domain are shown in blue. Two short sequences boxed outside of the Tg domain are the sites of the primer pair used to amplify the fragments of the Tg insertion sequence. 


\section{Conservation of Tg and MDIi-2 sequences}

The nucleic acid sequences of full-length MDIi-2 and $T g$ from Muscovy Duck were compared with those of other birds, including goose, quail, and chicken (GenBank accession No. goose, HM636053.1; quail, HQ130789.1; chicken, AY597054.1) (Table 2). The $T g$ sequences of Muscovy Duck shared over $95.77 \%$ identity with other birds, while MDIi-2 shared over $72.02 \%$ identity with $I-2$ from other birds.

Table 2. Similarity of nucleic acid and amino acid sequences of MDIi-2 and Tg domain among different birds (similarity, \%).

\begin{tabular}{lccc}
\hline Items for comparison & Ii-2 of goose & Ii-2 of quail & Ii-2 of chicken \\
\hline MDIi-2 & 88.79 & 74.39 & 72.02 \\
$T g$ of $M D I i-2$ & 96.83 & 95.77 & 95.77 \\
MDIi-2 & 94.74 & 74.83 & 72.51 \\
Tg of MDIi-2 & 96.83 & 96.83 & 96.83 \\
\hline
\end{tabular}

In comparison column, italics and upright letters indicate nucleic acid sequence and amino acid sequence, respectively, which were compared with those of goose, quail, and chicken. GenBank accession No. of Ii-2 cDNA of muscovy duck, goose, quail, and chicken are KF584915, HM636053.1, HQ130789.1, and AY597054.1 respectively. GenBank accession numbers of the amino acid sequences of Ii isoform containing Tg domain are: muscovy duck, AHB20201; goose, ADK91576.1; quail, ADM86479.1; chicken, AAT36346.1.

The open-reading frame of $M D I i-2$ encodes a 285 -amino acids protein (Figure 2) with a predicted molecular mass of $31.6 \mathrm{kDa}$ and a theoretical $\mathrm{pI}$ of 8.27 . The amino acid sequences of MDIi-2 contain 38 strong basic (+) amino acids (K, R), 35 strongly acidic (-) amino acids (D, E), 88 hydrophobic amino acids (A, I, L, F, W, V), and 72 polar amino acids (N, C, Q, S, T, Y). A single transmembrane domain was predicted from residues 28-56 in MDIi-2. The 63-amino acid Tg domain was found to be at $\mathrm{V}^{197}-\mathrm{S}^{259}$ in MDIi-2. Homology analysis demonstrated that the amino acid sequence of MDIi-2 shared remarkably high homology (72.51-94.74\%) with Ii-2 of other birds described above (Table 2). The closest relative to Muscovy Duck was goose (phylogenetic tree of Ii-2 in birds not shown). However, the amino acid sequence of the $\mathrm{Tg}$ domain was very similar to that of other birds (similarity of $96.83 \%$, Table 2). There were few amino acid differences among the Tg domain of all tested birds.

\section{Conservation in structure models of Tg domain among various species}

The 3-dimensional structure models of the Tg domain of Ii among the 4 birds (Figure 3) revealed that the backbone of Tg was remarkably similar in each model. There were some similar secondary structures, such as $1 \alpha$-helix, 6 linear regions, 10 turns, and a few ring areas of different lengths in all models. The linear regions, turns, and ring areas were nearly the same regarding their spatial conformations. The Tg structure models of Muscovy Duck and goose were very similar; however, those of quail and chicken were more similar than the former 2 species. Differences in the Tg structure models between the former 2 species and the latter 2 were based on the divergences of the $\alpha$-helix structure. The differences in the individual amino acids of the $\alpha$-helix may slightly disturb the helix framework. 

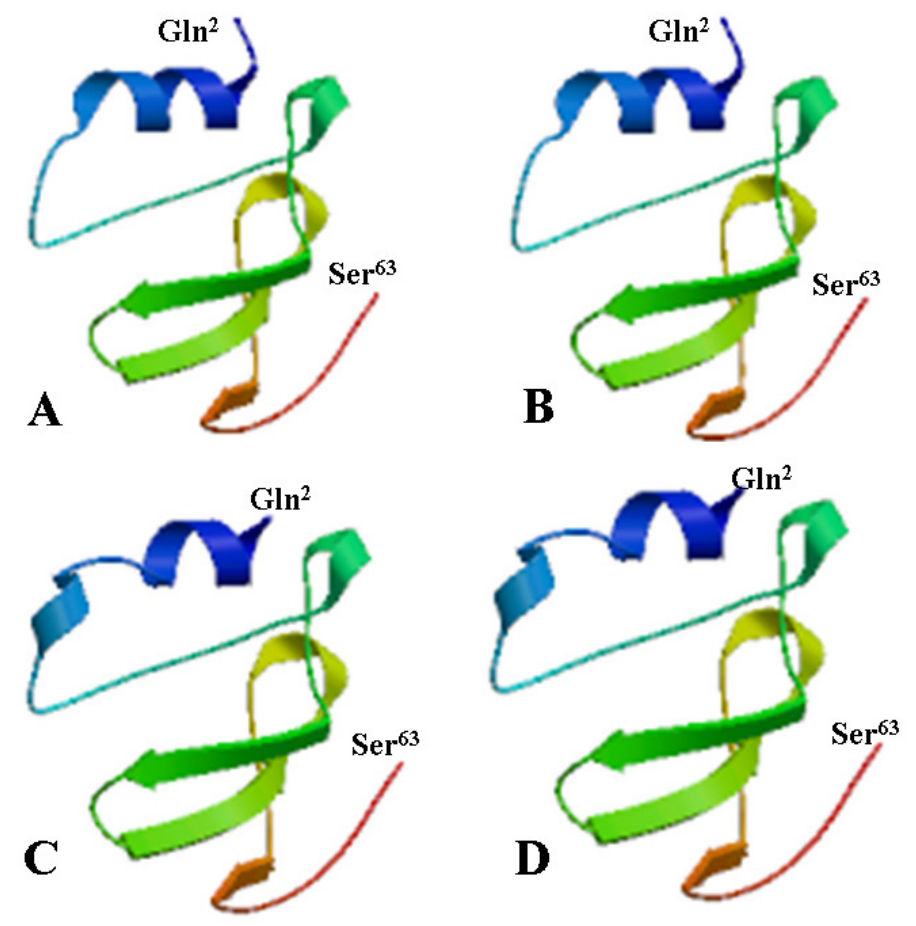

Figure 3. Three-dimensional structure models of Tg domain of Ii isoforms for 4 birds. The GenBank accession numbers of the amino acid sequences of Ii isoforms containing the Tg domain are: Muscovy Duck, AHB20201; goose, ADK91576.1; quail, ADM86479.1; chicken, AAT36346.1. The 3-D models of the Tg domain of Ii isoforms in Muscovy Duck (A), goose (B), quail (C), and chicken (D) were generated with the template 1icfI (2.00 A) of human Ii using the Swiss-Model server and were viewed using the RasMol software.

\section{MDIi-2 was expressed in all tissues but at different levels}

$M D I i-2$ expression was detected in various tissues, but expression levels were tissue-specific (Figure 4), similar to the expression pattern observed for MDIi-1 (Liu et al., 2011). MDIi-2 expression in the bursa fundus was the lowest of all tissues tested; thus, this level was selected as the calibrator. The highest expression of MDIi-2 was observed in spleen, which was 2.21-fold higher than in the calibrator. As a non-traditional immune tissue, $M D I i-2$ expression was 1.58 -fold higher in the intestinal mucosa than in the calibrator, and the level was nearly equal to the expression level in the bursa stipe at 1.54-fold $v s$ the calibrator. Thus, the spleen, intestinal mucosa, and bursa stipe showed the highest expression levels. The thymus, lung, and kidney abundantly expressed $M D I i-2$, all exceeding 1.10fold $v s$ the calibrator. In addition, the liver expressed $M D I i-2$ at a relatively lower level of approximately 0.79 -fold over the calibrator. Other tissues, such as the blood, brain, heart, and muscle, expressed $M D I i-2$ at similar but extremely low levels. Interestingly, $M D I i-2$ was also expressed at very high levels in the bursa stipe but extremely low levels in the bursa fundus. Thus, MDIi-2 expression was remarkably lower than that of MDIi-1 (Liu et al., 2011) in each tissue. 


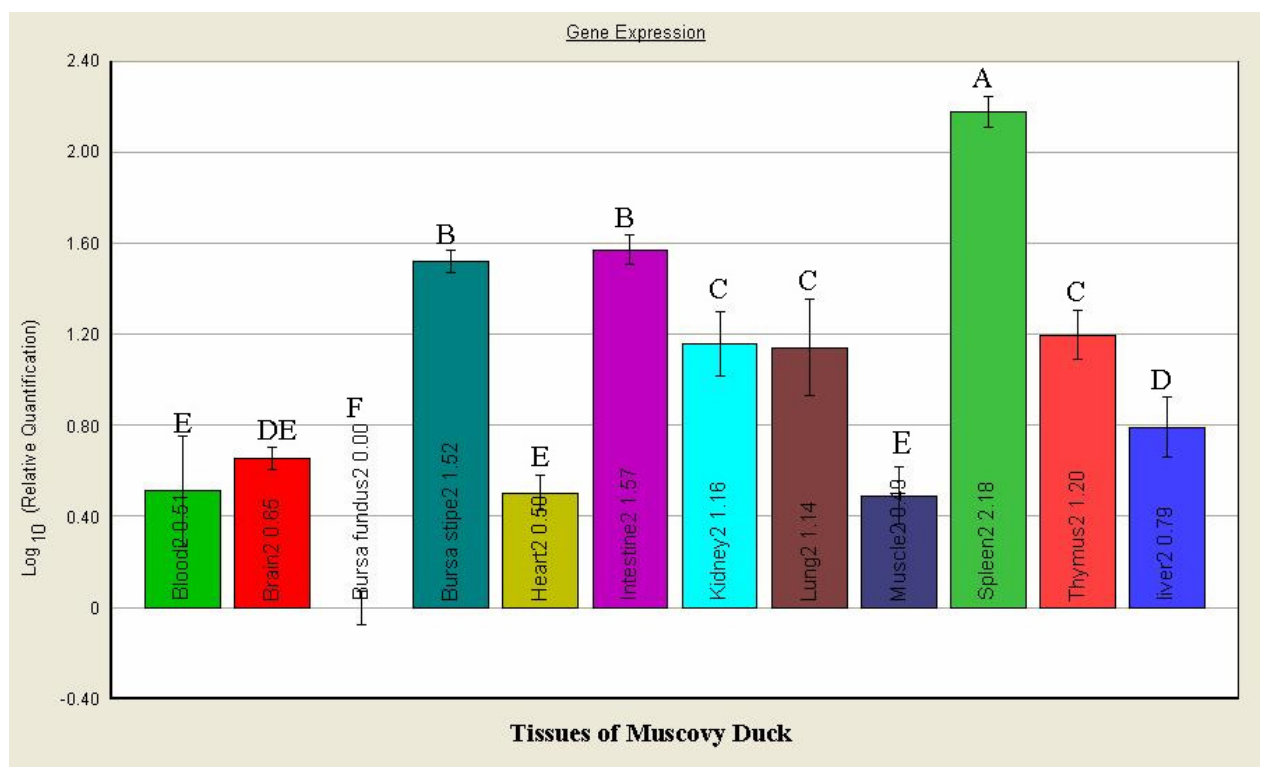

Figure 4. Expression profile of $M D I i-2$ in various tissues of Muscovy Duck. Expression levels were measured by real-time RQ-PCR in samples from the blood, brain, bursa fundus, bursa stipe, heart, intestinal mucosa, kidney, lung, muscle, spleen, thymus, and liver from Muscovy Ducks that were approximately 5 months of age. MDIi2 expression was normalized to the endogenous control $\beta$-actin. MDIi-2 expression in the bursa fundus served as a calibrator. MDIi-2 expression levels were achieved using the $2^{-\Delta \Delta C t}$ method of quantification. The relative expression level of MDIi-2 in different tissues is shown as the mean of 3 measurements and analyzed by 1-way analysis of variance. $\mathrm{P}<0.01$ is denoted with a capital letter above each bar. Groups marked with the same letters were not statistically different.

\section{DISCUSSION}

\section{Conservative Tg domain reflects rigorous alternative splicing of $\mathrm{MDIi}-2$}

This study demonstrated that the Tg domain is encoded in the MDIi gene. Previous results showed that the $\mathrm{Tg}$ domains of the Ii isoform in various birds, as well as in human and rat, were conserved (Zhong et al., 2006; Chen et al., 2011), as observed in our study. The conservative Tg domain is present in MDIi-1, so that MDIi-2 is expressed. Conservation of the Tg domain in MDIi is displayed in the following. First, Tg domains in various species are very high similar on the nucleic acid and amino acid levels according to sequence alignment (Zhong et al., 2006). Second, uniformity in the molecular structure of Tg was observed in structure modeling. Uniformity of molecular structure is ultimately determined by the amino acid sequence of the Tg domain. Third, the high level of consistency of insertion sites of the $\mathrm{Tg}$ domain in each Ii isoform also indicates the conservation of the Ii isoform and Tg domain in various species. High conservation of the Tg domain and nearly the same Tg insertion sites in the Ii isoforms imply rigorous and highly consistent mechanisms of alternative splicing during expression of Ii isoforms in various species (Chisa and Burke, 2007; Chen et al., 2011). These results agree with those of Chisa and Burke (2007), who revealed that mRNA splice-isoform selection is tightly controlled. 


\section{Conservation of MDIi-2 indicates the same function and similar evolution pressure among various species}

Proteins with similar molecular structures likely have similar functions (Bajaj and Bludell, 1984). The cysteine-rich Tg domain in p41 serves as protease inhibitor based on its unique folding structure and can cause protein hydrolysis in humans (Guncar et al., 1999; Reich et al., 2011), such that degradation of MHC II molecules during antigen-presentation is prevented (Bevec et al., 1996). Thus, the Ii isoforms containing the Tg domain in birds, including Muscovy Duck, may have common and essential roles as protease inhibitors during antigen-presentation. Protection of MHC II is also important in birds. As Ii has common functions among species, evolution has maintained the molecular structure of this protein. The tissue-specific expression of MDIi-2 may be related to its innate structure and essential function in each tissue (Ye et al., 2003; Liu et al., 2011). For example, MDIi-2 was expressed at very high levels in conventional immune organs such as the spleen, bursa stipe, and thymus. Although the intestinal mucosa and lungs are not considered to be immune organs, they expressed MDIi-2 at relatively high levels. This may be because the mucosa membrane of these 2 organs is located at a strategic position between the external environment and the most extended lymphoid tissue in organs that resist pathogens from the external environment (Mallegol et al., 2005).

The universality and identity of the Ii isoform is observed in variant invertebrate species, indicating its importance in biological evolutionary history. This suggests that similar survival pressures affect the evolution of Ii in different species. For example, pathogen invasion must be prevented in all species. This has been observed in the study of MHC II molecular evolution (Surridge et al., 2008; Evans and Neff, 2009).

\section{MDIi-2 is secondarily expressed to MDIi-1}

Our results demonstrated that the 2 forms of MDIi were coexpressed in each tissue, but the expression level of MDIi-2 was relatively lower than that of MDIi-1. The following observations support this hypothesis. First, 2 nucleic acid bands of approximately 100 and 290 $\mathrm{bp}$ were cloned using the same amplification system for the Tg fragment. However, the abundance of the 290-bp band containing the Tg domain was clearly lower than that of the 100-bp band. Second, differential expression of MDIi-1 and MDIi-2 in various tissues indicated the coexistence of $2 M D I i$ isoforms according to our real-time RQ-PCR results. The expression level of MDIi-2 (Figure 4) was consistently lower than that of MDIi-1 (Liu et al., 2011) in all tissues examined. Thus, MDIi-2 was coexpressed with MDIi-1, but MDIi-2 expression was secondary. Additionally, the expression pattern of the 2 forms of MDIi was same as observed in other species (Yamamoto et al., 1985; Zhong et al., 2006; Chen et al., 2011). Thus, the coexistence of the 2 forms of Ii illustrates the correlation between their functions in Muscovy Duck, similarly to in other species.

\section{CONCLUSION}

We cloned a 1377-bp cDNA sequence of MDIi-2 encoding a 285 -amino acid protein that contained a 63-amino acid insertion sequence of the Tg domain. The MDIi-2 and Tg 
domains showed high conservation, reflecting that MDIi-2 is rigorously alternatively spliced during expression, which is necessary for its function as a protease inhibitor during antigen presentation and expected based on similar survival pressures as other species, contributing to similar biological evolution. MDIi-2 was detected in all of the tissues tested at different levels, suggesting that MDIi-2 plays an essential role in each tissue and that tissue-specific expression may be related to the innate structures and essential functions of these tissues. The coexistence of MDIi-1 and MDII-2 illustrates that their functions are correlated in Muscovy Duck.

\section{ACKNOWLEDGMENTS}

Research supported by the Natural Scientific Research Major Projects (\#KJ2013A203) of the Anhui Department of Education and the National Natural Science Foundation of China (\#31172306).

\section{REFERENCES}

Arunachalam B, Lamb CA and Cresswell P (1994). Transport properties of free and MHC class II-associated oligomers containing different isoforms of human invariant chain. Int. Immunol. 6: 439-451.

Bajaj M and Blundell T (1984). Evolution and the tertiary structure of proteins. Annu. Rev. Biophys Bioeng. 13: 453-492. Bergmann H (2012). Business as usual: the $\mathrm{p} 35$ isoform of human CD74 retains function in antigen presentation. Immunol. Cell Biol. 90: 839-840.

Bevec T, Stoka V, Pungercic G, Dolenc I, et al. (1996). Major histocompatibility complex class II-associated p41 invariant chain fragment is a strong inhibitor of lysosomal cathepsin L. J. Exp. Med. 183: 1331-1338.

Chen FF and Yu WY (2010). A new type immune carrier - invariant chain of MHC II. Chin. J. Immunol. 26: 573-576.

Chen F, Pan L, Dai Y, Ye H, et al. (2011). Characteristics of expression of goose invariant chain gene and comparison of its structure among different species. Poult. Sci. 90: 1664-1670.

Chen F, Wu C, Pan L, Xu F, et al. (2013). Cross-species association of quail invariant chain with chicken and mouse MHC II molecules. Dev. Comp. Immunol. 40: 20-27.

Chisa JL and Burke DT (2007). Mammalian mRNA splice-isoform selection is tightly controlled. Genetics 175: 10791087.

Criscitiello MF, Ohta Y, Graham MD, Eubanks JO, et al. (2012). Shark class II invariant chain reveals ancient conserved relationships with cathepsins and MHC class II. Dev. Comp. Immunol. 36: 521-533.

Evans ML and Neff BD (2009). Major histocompatibility complex heterozygote advantage and widespread bacterial infections in populations of Chinook salmon (Oncorhynchus tshawytscha). Mol. Ecol. 18: 4716-4729.

Gedde-Dahl M, Freisewinkel I, Staschewski M, Schenck K, et al. (1997). Exon 6 is essential for invariant chain trimerization and induction of large endosomal structures. J. Biol. Chem. 272: 8281-8287.

Guncar G, Pungercic G, Klemencic I, Turk V, et al. (1999). Crystal structure of MHC class II-associated p41 Ii fragment bound to cathepsin $\mathrm{L}$ reveals the structural basis for differentiation between cathepsins $\mathrm{L}$ and $\mathrm{S}$. EMBO J. 18: 793803.

Holst PJ, Christensen JP and Thomsen AR (2011). Vaccination against lymphocytic choriomeningitis virus infection in MHC class II-deficient mice. J. Immunol. 186: 3997-4007.

Hung CF, Tsai YC, He L and Wu TC (2007). DNA vaccines encoding Ii-PADRE generates potent PADRE-specific CD4+ T-cell immune responses and enhances vaccine potency. Mol. Ther. 15: 1211-1219.

Landsverk OJ, Bakke O and Gregers TF (2009). MHC II and the endocytic pathway: regulation by invariant chain. Scand. J. Immunol. 70: 184-193.

Liu SJ, Chen FF, Dai Y, Wu C, et al. (2011). Molecular characterization and tissue-specific expression of invariant chain in the muscovy duck (Cairina moschata). Genet. Mol. Res. 10: 2867-2880.

Mallegol J, van Niel G and Heyman M (2005). Phenotypic and functional characterization of intestinal epithelial exosomes. Blood Cells Mol. Dis. 35: 11-16.

Nagaraj S, Neumann J, Winzen B, Frank S, et al. (2008). Pancreas carcinoma antigen fused to invariant chain elicits T-cell response and tumor growth inhibition. Pancreas 37: 321-327.

O’Sullivan DM, Noonan D and Quaranta V (1987). Four Ia invariant chain forms derive from a single gene by alternate 
splicing and alternate initiation of transcription/translation. J. Exp. Med. 166: 444-460.

Reich M, Zou F, Sienczyk M, Oleksyszyn J, et al. (2011). Invariant chain processing is independent of cathepsin variation between primary human B cells/dendritic cells and B-lymphoblastoid cells. Cell Immunol. 269: 96-103.

Strubin M, Berte C and Mach B (1986). Alternative splicing and alternative initiation of translation explain the four forms of the Ia antigen-associated invariant chain. EMBO J. 5: 3483-3488.

Surridge AK, van der Loo W, Abrantes J, Carneiro M, et al. (2008). Diversity and evolutionary history of the MHC DQA gene in leporids. Immunogenetics 60: 515-525.

Wälchli S, Kumari S, Fallang LE, Sand KM, et al. (2014). Invariant chain as a vehicle to load antigenic peptides on human MHC class I for cytotoxic T-cell activation. Eur. J. Immunol. 44: 774-784.

Walline CC, Deffit SN, Wang N, Guindon LM, et al. (2014). Virus-encoded ectopic CD74 enhances poxvirus vaccine efficacy. Immunology 141: 531-539.

Warmerdam PA, Long EO and Roche PA (1996). Isoforms of the invariant chain regulate transport of MHC class II molecules to antigen processing compartments. J. Cell Biol. 133: 281-291.

Xu FZ, Dai Y, Li LM, Ding XL, et al. (2013). Molecular cloning and site-directed mutagenesis of leucine-based sorting motifs of the porcine invariant chain. Genet. Mol. Res. 12: 4489-4499.

Yamamoto K, Floyd-Smith G, Francke U, Koch N, et al. (1985). The gene encoding the Ia-associated invariant chain is located on chromosome 18 in the mouse. Immunogenetics 21: 83-90.

Ye Q, Finn PW, Sweeney R, Bikoff EK, et al. (2003). MHC class II-associated invariant chain isoforms regulate pulmonary immune responses. J. Immunol. 170: 1473-1480.

Zhong D, Yu W, Bao M, Xu Z, et al. (2006). Molecular cloning and mRNA expression of duck invariant chain. Vet. Immunol. Immunopathol. 110: 293-302. 